九州大学学術情報リポジトリ

Kyushu University Institutional Repository

\title{
NONPARAMETRIC INFERENCES WITH A PRELIMINARY TEST
}

Tamura, Ryoj i

Shimane University

https://doi.org/10.5109/13016

出版情報: 統計数理研究. 11 (3/4)，pp. 39-61，1965-03. Research Association of Statistical Sciences

バージョン :

権利関係 : 


\title{
NONPARAMETRIC INFERENCES WITH A PRELIMINARY TEST
}

\author{
By \\ Ryoji TAMURa \\ (Received February 1, 1964)
}

§1. Introduction. This paper is concerned with certain successive process of statistical inferences in the nonparametric circumstance. The notions of such successive process have been developed in the normal theorey by Paul [8], Kitagawa [5] and others. However it seems that any result along this line has not ever been appeared in the nonparametric case up to the present time. We shall here deal with two types of the nonparametric problem. One of them is concerned with one sample problem (A) and other is two sample problem (B).

(A) Let $X_{1}, X_{2}, \cdots, X_{m}$ and $Y_{1}, Y_{2}, \cdots \cdots, Y_{n}$ be two samples from the continuous distributions $F_{1}(x)$ and $F_{2}(y)$ respectively. Our "sometimes pool" one sample problem of the $T T$ type may be, without loss of generality, for.nulated a; follows. The main object is to test the hypothesis

$$
H: F_{1}(0)=1 / 2
$$

against the alternative

$$
H^{\prime}: F_{1}(0)=p(>1 / 2) \text {. }
$$

There exist some cases where it may be more effective to consider in connection with the knowledge from $Y$ sample. Thus we shall first test the hypothesis

$$
H_{1}: F_{1}(x) \equiv F_{2}(x)
$$

against the alternative

$$
H_{1}^{\prime}: F_{1}(x)>F_{2}(x)
$$

and then decide how to use $Y$ sample. The main test is performed by Sign test and the preliminary test is by Wilcoxon test. In section 2, we shall investigate the behaviour of the size and power function of the test when sample sizes are large and section 3 is concerned with small sample. The $T E$ type problem about the median of $X$ will be considered in section 4 .

(B) Let $X_{1}, X_{2}, \cdots \cdots, X_{m_{1}} ; Y_{1}, Y_{2}, \cdots \cdots, Y_{m_{2}}$ and $Z_{1}, Z_{2}, \cdots \cdots, Z_{m_{3}}$ be three samples of size $m_{1}, m_{2}, m_{3}$ from the continuous distribution functions $F_{1}(x), F_{2}(y)$ and $F_{3}(z)$ respectively. Though it is our main purpose to test 
the hypothesis

$$
K: F_{1}(x) \equiv F_{2}(x)
$$

against the alternative

$$
K^{\prime}: F_{1}(x)>F_{2}(x),
$$

we shall consider to test the hypothesis as a preliminary step

$$
K_{1}: F_{2}(x) \equiv F_{3}(x)
$$

against the alternative

$$
K^{\prime}{ }_{1}: F_{2}(x)>F_{3}(x)
$$

from the same reason as in (A). Wilcoxon statistics will be utilized for these two sample tests. The size of test and power properties in the large sample case will be considered in section 5. Some discussions will be developed in section 6 about the test statistics and the alternatives.

\section{$\S 2$. One sample problem of the $T T$ type- The large sample case -}

2. 1. Test procedure. Define the following U-statistics as the each test statistic,

$$
\begin{gathered}
U_{1}=\frac{1}{m n} \sum_{i=1}^{m} \sum_{j=1}^{n} \phi\left(X_{i}, X_{j}\right), \\
U_{2}=\frac{1}{m} \sum_{i=1}^{m} \phi\left(X_{i}\right), \\
U_{3}=\frac{1}{N}\left\{\sum_{i=1}^{m} \phi\left(X_{i}\right)+\sum_{j=1}^{n} \phi\left(Y_{j}\right)\right\}, N=m+n,
\end{gathered}
$$

where $\phi(x, y)=1(0)$ for $x<y$ (otherwise) and $\phi(x)=1(0)$ for $x>0$ (otherwise).

The statistic $U_{1}$ is what is called as Wilzoxon's statistic (or MannWhitney's) that is used at the preliminary test and the other U's are in Sign tests.

Now we adopt the following test procedure where we reject the hypothesis $H$ when either the proposition (I) or (II) holds

$$
\begin{aligned}
& \text { (I) } \quad U_{1} \geqq u_{\alpha_{1}} \text { and } U_{2} \geqq u_{\alpha_{2}}, \\
& \text { (II) } U_{1}<u_{\alpha_{1}} \text { and } U_{3} \geqq u_{\alpha_{3}} .
\end{aligned}
$$

The constants $u_{\alpha_{i}}$ may be determined by (4), (5) from the well-known fact that the asymptotic distribution of these U-statistics is normal. 


$$
\begin{gathered}
u_{\alpha_{1}}=\frac{1}{2}+\frac{z_{\alpha_{1}}}{V N} \sqrt{12\left(\frac{1}{\lambda}+\frac{1}{1-\lambda}\right)}, m / N=\lambda, \\
u_{\alpha_{2}}=\frac{1}{2}+\frac{z_{\alpha_{2}}}{2 \sqrt{m}}, \quad u_{\alpha_{3}}=\frac{1}{2}+\frac{z_{\alpha_{3}}}{2 / N}, \\
1-\Phi\left(z_{\alpha_{i}}\right)=\alpha_{i}, \Phi(x)=\frac{1}{V 2 \pi} \int_{-\infty}^{x} \exp \left(-t^{2} / 2\right) d t=\int_{-\infty}^{x} \varphi(t) d t,
\end{gathered}
$$

where $\alpha_{i}$ is the size of each partial test in the preliminary or main step. From the test procedure (I) and (II), the size $\alpha$ and power function $\beta(p)$ of the test may be formally written as follows,

(6) $\alpha=\operatorname{Pr}\left(U_{1} \geqq u_{\alpha_{1}}\right.$ and $\left.U_{2} \geqq u_{\alpha_{2}} \mid H\right)+\operatorname{Pr}\left(U_{1}<u_{\alpha_{1}}\right.$ and $\left.U_{3} \geq u_{\alpha_{3}}{ }^{\prime} H\right)$,

(7) $\beta(p)=\operatorname{Pr}\left(U_{1} \geqq u_{\alpha_{1}}\right.$ and $\left.U_{2} \geqq u_{\alpha_{2}} H^{\prime}\right)+\operatorname{Pr}\left(U_{1}<U_{\alpha_{1}}\right.$ and $\left.U_{3} \geq u_{\alpha_{3}} \mid H^{\prime}\right)$.

2.2 The asymptotic distribution. The parameters relating to the distributions of the statistics $U$ may be easily computed as follows,

$$
E\left(U_{1}\right)=\int_{-\infty}^{\infty} F_{1} d F_{2}=\mu, E\left(U_{2}\right)=p, E\left(U_{3}\right)=\lambda p+(1-\lambda) F_{2}(0),
$$

(8) $\operatorname{Var} U_{1}=\frac{1}{m}\left(r-\mu^{2}\right)+\frac{1}{n}\left(q-\mu^{2}\right)=\sigma_{1}^{2}, \operatorname{Var} U_{2}=\frac{1}{m} p(1-p)=\sigma_{2}^{2}$,

$$
\operatorname{Var} U_{3}=\frac{\lambda}{N} p(1-p)+\frac{1-\lambda}{N} F_{2}(0)\left\{1-F_{2}(0)\right\}=\sigma_{3}^{2},
$$

where

$$
q=\int_{-\infty}^{\infty} F_{1}^{2} d F_{2}, r=\int_{-\infty}^{\infty}\left(1-F_{2}\right)^{2} d F_{1} \text { and } F_{i}=F_{i}(x) .
$$

As for the correlation of U's, we get

$$
\begin{aligned}
& \rho\left(U_{1}, U_{2}\right)=\left[\int_{-\infty}^{0} F_{1} d F_{2}+p\left\{1-F_{2}(0)\right\}-\mu p\right] / m \sigma_{1} \sigma_{2}=\rho_{2}, \\
& \rho\left(U_{1}, U_{3}\right)=\left[2 \int_{-\infty}^{0} F_{1} d F_{2}+p\left\{1-F_{2}(0)\right\}-\mu p-\mu F_{2}(0)\right] / N \sigma_{1} \sigma_{3}=\rho_{3} .
\end{aligned}
$$

It has been shown by Hoeffding [3] that the joint distribution of $g$ U-statistics, which are constructed only within one sample, is asymptotically normal. We must extend this theorem for our generalized U-statistics. We consider only the joint distribution of $U_{1}$ and $U_{2}$, for the other may be dealt similarly. Define the statistic

$$
V_{N}=\frac{1}{V \lambda m} \sum_{i=1}^{m}\left\{\psi_{10}\left(X_{i}\right)-\mu\right\}+\frac{1}{V(1-\lambda) n} \sum_{j=1}^{n}\left\{\psi_{01}\left(Y_{j}\right)-\mu\right\}=V_{1}+V_{2},
$$


where

$$
\begin{aligned}
& \psi_{10}(x)=E \phi(x, Y)=1-F_{2}(x), \\
& \psi_{01}(y)=E \phi(X, y)=F_{1}(y) .
\end{aligned}
$$

Then it has been shown by Dwass [2] that $V_{N}$ is equivalent to $/ \bar{N}\left(U_{1}-\mu\right)$ in the sense that

$$
E\left[V N\left(U_{1}-\mu\right)-V_{N}\right]^{2} \rightarrow 0 \text { as } \boldsymbol{N} \rightarrow \infty
$$

and they have a same asymptotically normal distribution. Since it holds

$$
\text { 1/m(U }\left(U_{2}-p\right)=\frac{1}{v} \sum_{i=1}^{m}\left\{\phi\left(X_{i}\right)-p\right\},
$$

we may construct the following random vectors

$$
\mathrm{W}_{j}=\left(\phi\left(X_{j}\right)-p, \frac{1}{\sqrt{\lambda}} \psi_{10}\left(X_{j}\right)\right), j=1,2, \cdots \cdots, m .
$$

Since $m W_{j}^{\prime}$ s are independent and identically distribubuted, the statistic $\sum_{j=1}^{m} W_{j} / m$ is asymptotically distributed as a bivariate normal distribution by the Central Limit Theorem if having the finite second order moment not all zero. Noticing that the statistic $V_{2}$ is independent on both the statistics $V_{\text {: }}$ and $U_{2}$, we may show after some computations that the asymptotic discribution of the statistic $\left(U_{1}-\mu\right) / \sigma_{1}$ and $\left(U_{2}-p\right) / \sigma_{2}$ is the bivariate normal distribution with zero means, unit variances and correlation $\rho_{2}$. A similar result may be also established for $U_{1}$ and $U_{3}$. More generally, it can be extended for the similar $g$ U-statistics.

2.3. The size of the test. From the considerations in 2.2, the size $\alpha$ may be asymptotically expressed as follows,

$$
\alpha=\int_{k_{0 N}}^{\infty} \int_{z_{\alpha 2}}^{\infty} g\left(u, v ; 0,1, \rho_{2,0}\right) d u d v+\int_{-\infty}^{k_{0 N}} \int_{k_{0 N}^{\prime}}^{\infty} g\left(u, v ; 0,1, \rho_{3,0}\right) d u d v,
$$

where $g(u, v ; 0,1, \rho)$ is the bivariate normal density with zero means, unit variances and correlation $\rho$ and

$$
\begin{aligned}
& k_{0 N}=\left(u_{\alpha_{1}}-\mu\right) / \sigma_{1,0}, \\
& k_{0 N}^{\prime}=\left(u_{\alpha_{3}}-\frac{\lambda}{2}-(1-\lambda) F_{2,0}(0)\right) / \sigma_{3,0} .
\end{aligned}
$$

$F_{2,0}, \sigma_{3,0}$ and $\rho_{i, 0}$ are respectively the value under the hypothesis $H$. Now we assume that $F_{2}(x)$ be the Lehmann type alternative

$$
F_{2}(x)=F_{1}^{1+\theta}(x), \theta \geqq 0 \text {. }
$$

As Lehmann [6] has appointed, this assumption is very effective in the case where the purpose is in comparision between some nonparametric tests. 
We denote the size of the test by $\alpha(\theta)$.

(i) The case $\theta=\theta_{0}$ ( $\theta_{0}$ is any positive constant). Since $k_{O N} \rightarrow-\infty$ as $N \rightarrow \infty$ from (13), we get from (12)

$$
\lim _{N \rightarrow \infty} \alpha\left(\theta_{0}\right)=\int_{-\infty}^{\infty} \int_{z_{\alpha_{2}}}^{\infty} g\left(u, v ; 0,1, \rho_{2,0}\right) d u d v=\alpha_{2} .
$$

Thus it has been proved that the size $\alpha\left(\theta_{0}\right)$ tends to the value $\alpha_{2}$ of the size of the "never pool" Sign test.

(ii) $\theta=\theta_{N}=r / v \bar{N}(r \geq 0)$. We may get from (8) and (14),

$$
\mu\left(\theta_{N}\right)=\frac{1}{2}+\frac{r}{4} / \bar{N}+o(1 / v / \bar{N})
$$

$$
\begin{aligned}
& F_{2}(0)=\frac{1}{2}-\frac{r}{2 \gamma} \log 2+o(1 / 1 / N), \\
& \rho_{2}=V(1-\lambda) / 4 \sqrt{p(1-p)}+o(1 / V \bar{N}), \rho_{3}=o(1 / \sqrt{N}) \text {. } \\
& \rho_{2,0}=\sqrt{3(1-\lambda)} / 2
\end{aligned}
$$

and from (13)

$$
\begin{aligned}
& \lim _{N \rightarrow \infty} k_{O N}=z_{\alpha 1}-r / 4 / \frac{1}{12}\left(\frac{\left.1+\frac{1}{\lambda}\right)}{1-\lambda}\right)\left(=k_{0}\right), \\
& \lim _{N \rightarrow \infty} k_{O N}^{\prime}=z_{\alpha_{3}}+(1-\lambda) r \log 2\left(=k_{0}^{\prime}\right) .
\end{aligned}
$$

Using (16) and (17),

$$
\alpha\left(\theta_{N}\right)=\int_{k_{0}}^{\infty} \int_{z_{\boldsymbol{\alpha}_{2}}}^{\infty} g\left(u, v ; 0,1, \frac{\overline{3(1-\lambda)}}{2}\right) d u d v+\Phi\left(k_{0}\right)\left\{1-\Phi\left(k_{0}^{\prime}\right)\right\} .
$$

In order to compute the value of $\alpha\left(\theta_{N}\right)$, we may use Owen's table [7] which is relating to the computations of bivariate probabilities. Table I and Figure I are the results for each value of $\alpha_{1}$ when $\alpha_{2}=\alpha_{3}=0.0495$ and $\lambda=1 / 2$.

TABLE I The size of the test

\begin{tabular}{c|ccccc}
\hline \multicolumn{1}{c|}{$r$} & 0 & $\sqrt{3}$ & $2 \sqrt{ } 3$ & $3 \sqrt{ } 3$ & $4 \sqrt{3}$ \\
\hline$\alpha_{1}$ & & & & \\
.0495 & .0628 & .0431 & .0464 & .0490 & .0494 \\
.0968 & .0685 & .0487 & .0493 & .0494 & .0495 \\
.1587 & .0726 & .0511 & .0494 & .0495 & .0495 \\
.2119 & .0743 & .0528 & .0496 & .0495 & .0495 \\
.3085 & .0750 & .0528 & .0497 & .0495 & .0495 \\
.5 & .0712 & .0520 & .0496 & .0495 & .0495 \\
.6915 & .0640 & .0507 & .0495 & .0495 & .0495 \\
.9115 & .0538 & .0497 & .0495 & .0495 & .0495 \\
\hline
\end{tabular}






FIGURE I

2.4. The properties of the power. From the fact that the joint distribution of our U-statistics is asymptotically normal, the power function $\beta(p)$ in (7) may be expressed as follows,

(19) $\beta(p)=\int_{k_{0 N}}^{\infty} \int_{k_{1 N}}^{\infty} g\left(u, v ; 0,1, \rho_{2}\right) d u d v+\int_{-\infty}^{k_{0 N}} \int_{k_{2 N}}^{\infty} g\left(u, v ; 01, \rho_{3}\right) d u d v$, where

$$
\begin{aligned}
& k_{1 N}=\left(u_{\alpha_{2}}-p\right) / \sigma_{2}, k_{0 N}=z_{\alpha_{1}}-\sqrt{3 \lambda(1-\lambda)} / 2, \\
& k_{2 N}=\left(u_{\alpha_{3}}-\lambda p-(1-\lambda) F_{2}(0)\right) / \sigma_{3} .
\end{aligned}
$$

(i) The case $p=p_{0}$ ( $p_{0}$ is any constant larger than $1 / 2$ ). If the value of $\theta$ equals to any positive constant $\theta_{0}$, the values of all $k$ 's approach $-\infty$ as $N \rightarrow \infty$ from (17) and (20). Hence

$$
\lim _{N \rightarrow \infty} \beta\left(p_{0}\right)=\int_{-\infty}^{\infty} \int_{-\infty}^{\infty} g\left(u, v ; 0,1, \rho_{2}\right) d u d v=1 .
$$

If $\theta=\theta_{N}=r / \sqrt{N}(r \geqq 0)$, then $k_{0 N} \rightarrow k_{0}$ and other $k_{i N} \rightarrow-\infty$. Thus

$$
\lim _{N \rightarrow \infty} \beta\left(p_{0}\right)=\int_{k_{0}}^{\infty} \varphi(u) d u+\int_{-\infty}^{k_{0}} \varphi(u) d u=1 .
$$

In either case, the value of the power function $\beta(p)$ tends to unity as $N$ approaches infinity when $p=p_{0}$. It is well known that the usual Sign test has also the same property of consistency, so that we must investigate the 
behaviour of large sample powers in the neighbourhood of $p=1 / 2$.

(ii) The case $p=p_{m}=\frac{1}{2}+\frac{\delta}{\sqrt{\boldsymbol{m}}}(\delta<0)$. Let us assume the alternative

$$
p=p_{m}=\frac{1}{2}+\frac{\delta}{\sqrt{m}}, \delta>0
$$

and derive the relative asymptotic efficiency of the "sometimes pool" test with regard to the "never pool" Sign test. It may be defined as the limit value of the reciprocal ratio of the sizes of $X$ samples necessary to achieve the same power against the same alternative at the same significance level. The power of the "never pool" Sign test with the size $\alpha$ of the test and the sample size $M$ is asymptotically expressed by the form

$$
\beta_{1}(p)=1-\Phi\left(\frac{\frac{1}{2}+\frac{z_{\alpha}}{2 / \bar{M}}-p}{\vee p(1-p) / \bar{M}}\right)
$$

After some computations containing power series expansions about $p=1 / 2$ of the power functions, we may get the asymptotic efficiency $\boldsymbol{e}_{s, n}$ as follows,

$$
\left.e_{s, n}=\frac{1}{\varphi\left(z_{\alpha}\right)^{2}} \Gamma_{-} \varphi\left(k_{1}\right)\left\{1-\Phi\left(\frac{k_{0}-\rho_{2,0} k_{1}}{\sqrt{1-\rho_{2,0}^{2}}}\right)\right\}+\frac{1}{\sqrt{\lambda}} \varphi\left(k_{2}\right) \Phi\left(\frac{k_{0}-\rho_{3,0} k_{2}}{\sqrt{1-\rho_{3,0}^{2}}}\right)\right]^{2}
$$

where $k_{i}=\lim _{N \rightarrow \infty} k_{i N}$.

In the case $\theta=\theta_{0}$ (positive constant), we get $e_{s, n}=1$, for $k_{0}=-\infty, k_{1}=z_{\alpha_{2}}$ and moreover $\alpha=\alpha_{2}$ from (15).

Lastly we must investigate the behaviour of $e_{s, n}$ in the case $\theta=\theta_{N}=r / \sqrt{N}$. Under $\lambda=1 / 2$,

$$
\begin{aligned}
& k_{0}=z_{\alpha_{1}}-\frac{\sqrt{3}}{4} \gamma+O(1 / \sqrt{N}), k_{1}=z_{\alpha_{2}}+O(1 / \sqrt{N}), \\
& k_{2}=z_{\alpha_{3}}+\frac{\gamma}{2} \log 2+O(1 / \sqrt{N}), \\
& \rho_{2,0}=\sqrt{6} / 4+O(1 / v N), \rho_{3,0}=O(1 / \sqrt{N}) .
\end{aligned}
$$

We may rewrite the expression (25)

$$
\begin{aligned}
e_{s, n} & =\frac{1}{\varphi\left(z_{\alpha}\right)^{2}}\left[\varphi\left(z_{\alpha_{2}}\right)\left\{1-\Phi\left(\frac{z_{\alpha_{1}}-\frac{\sqrt{3}}{4} \gamma-\frac{\sqrt{6}}{4} z_{\alpha_{2}}}{\sqrt{10 / 4}}\right)\right\}\right. \\
& \left.+\sqrt{2} \varphi\left(z_{\alpha_{3}}+\frac{\gamma \log 2}{2}\right) \Phi\left(z_{\alpha_{1}}-\frac{\sqrt{3}}{4} \gamma\right)\right]^{2}
\end{aligned}
$$

We may get Table II by using the values of $\alpha$ in Table I in the case $\alpha_{2}=$ $\alpha_{3}=0.0494$. 
TABLE II Asymptotic Efficiency

\begin{tabular}{cccccc}
\hline$\alpha_{1}$ & 0 & $\sqrt{ } 3$ & $2 \sqrt{3}$ & $3 \sqrt{ } 3$ & $3 \sqrt{ } 3$ \\
\hline .0495 & 1.656 & 1.041 & .927 & .982 & 1 \\
.0968 & 1.602 & 1.088 & .960 & .994 & 1 \\
.2119 & 1.566 & 1.122 & 1.011 & 1 & 1 \\
.3085 & 1.542 & 1.112 & 1.012 & 1 & 1 \\
.5 & 1.459 & 1.090 & 1.007 & 1 & 1 \\
.6915 & 1.323 & 1.049 & 1.004 & 1 & 1 \\
\hline
\end{tabular}

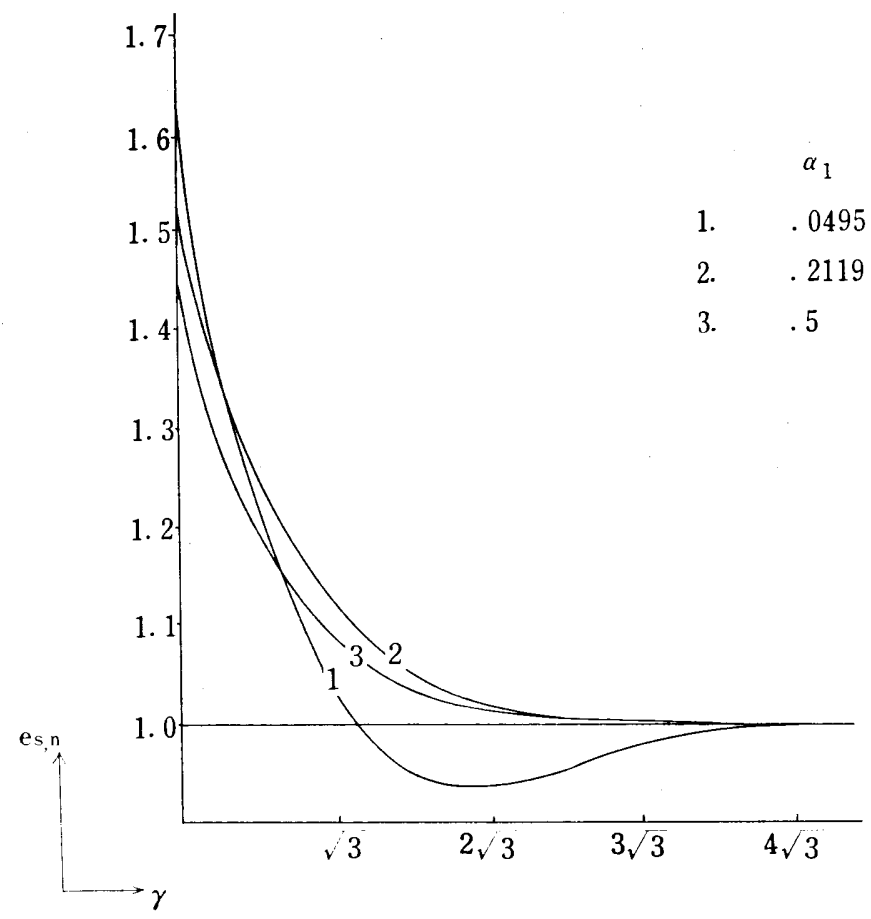

FIGURE II

\section{§3. Small sample case.}

3. 1. Test procedure. In the previous section, we have obtained some asymptotic results about the size and power of the "sometimes pool" test in the large sample case. We shall in this section inquire about the small sample case. We modify the notations slightly. Let $U_{1}$ be the rank sum of the $X$ sample in the combined sample and $U_{2}$ and $U_{3}$ be respectively $m$ $U_{2}$ and $N U_{3}$ in the previous section. There exist $(m+n) ! / m ! n !$ total orderings of $X$ and $Y$ samples. Let $\lambda_{\alpha_{1}}$ be such number that the ratio of the number of the orderings satisfying the relation $U_{1} \leqq \lambda_{\alpha}$. to $(m+n) ! / m$ ! $n$ ! is equal to $100 \alpha_{1} \%$ and other $\lambda_{\alpha_{i}}$ be determined by

$$
\sum_{j=\lambda_{\alpha_{2}}}^{m}\left(\begin{array}{c}
m \\
j
\end{array}\right) 2^{-m}=\alpha_{2}, \sum_{j=\lambda_{\alpha_{3}}}^{N}\left(\begin{array}{l}
N \\
j
\end{array}\right) 2^{-N}=\alpha_{3} .
$$


Then the test procedure is to reject the hypothesis $H$ when (I) or (II) holds,

$$
\begin{aligned}
& \text { (I) } U_{1} \leqq \lambda_{\alpha_{1}} \text { and } U_{2} \geq \lambda_{\alpha_{2}}, \\
& \text { (II) } \quad U_{1}>\lambda_{\alpha_{1}} \text { and } U_{3} \geq \lambda_{\alpha_{3}} .
\end{aligned}
$$

We cannot, in general, express the above procedure in any convenient form to compute and hence we are compelled to consider separately for each value of $m, n$ and $a_{i}$.

Let $\pi_{\lambda, \mu}$ be the total orderings of all $X^{\prime} s$ and $Y^{\prime} s$ in consideration where only the largest $\lambda+\mu$ variables (contain $\lambda X^{\prime}$ s and $\mu Y^{\prime}$ s) are held in fixed order and all are less than zero and $x \pi_{\lambda, \mu}$ be such $\pi_{\lambda, \mu}$ that the $(N-\lambda-\mu)$ th smallest is a $X . P_{m, n}^{\pi_{\lambda, \mu}}$ and $P_{m, n}^{x \pi_{\lambda, \mu}}$ be their probabilities. The same are also defined for $y \pi_{\lambda, \mu}$. Let $P_{m, n}$ be the probability that all $m X^{\prime}$ s and $n Y^{\prime}$ s are smaller than zero. Under the Lehmann type alternative $F_{2}=F_{1}^{1+\theta}$, we may derive the values of the probabilities

$$
\begin{aligned}
& P_{m, n}=p^{m+n(1+\theta)}, \\
& P_{m, n}^{x \pi_{\lambda, \mu}}=(m-\lambda) P_{m, n}^{\pi_{\mu, \mu}} /\{(m-\lambda)+(n-\mu)(1+\theta)\}, \\
& P_{m, n}^{j \pi \pi_{\lambda, \mu}}=(n-\mu)(1+\theta) P_{m, n}^{\pi_{\lambda, \mu}} /\{(m-\lambda)+(n-\mu)(1+\theta)\} .
\end{aligned}
$$

As a special case,

$$
\begin{aligned}
& P_{m, n}^{x}=m p^{m+n(1+\theta)} /\{m+n(1+\theta)\}, \\
& P_{m, n}^{y}=n(1+\theta) p^{m+n(1+\theta)} /\{m+n(1+\theta)\} .
\end{aligned}
$$

These formulas make the numerical computations of the power simple slightly.

3. 2. The power function. Now consider the simple case that $m=3$, $n=4, \alpha_{1}=0.2, \alpha_{2}=1 / 8$ and $\alpha_{3}=1 / 16$. The critical region of the preliminary test may be consisted of the following 7 orderings among the total 35 ,

$$
\begin{aligned}
& (x x x y y y y),(x x y x y y y),(x y x x y y y),(x x y y x y y), \\
& (y x x x y y y),(x y x y x y y),(x x y y y x y) .
\end{aligned}
$$

The orderings which lie in the critical region of the test may be rumbered by 81 . Therefore it is seemingly to trouble to compute the values of such probabilities, but we may make the computation very easy by combinating them suitably. The case above becomes the following form,

$$
\begin{aligned}
\beta(p) & =P_{3,4}+\operatorname{Pr}(\text { any one } Y>0) \cdot P_{3,3}+\operatorname{Pr}(\text { any one } X>0) \cdot P_{2,4} \\
& +\operatorname{Pr}\left(\text { all } Y^{\prime} \mathrm{s}>0\right) \cdot P_{3,0}+\operatorname{Pr}\left(\text { any three } Y^{\prime} \mathrm{s}>0\right) \cdot P_{3,1} \\
& +\operatorname{Pr}\left(\text { any two } Y^{\prime} \mathrm{s}>0\right)\left\{P_{3,2}^{y}+\operatorname{Pr}\left(x x y y x_{0}\right)+\operatorname{Pr}(x y x y x 0)\right\} .
\end{aligned}
$$


Thus the power function is given by

$$
\beta(p)=p^{3}\left\{1+3\left(\frac{1}{p}-1\right) p^{4(1+\theta)}-18 p^{2(1+\theta)}\left(1-p^{1+\theta}\right)^{2}(2+\theta)^{2} \cdot(3+\theta) \cdot(5+2 \theta)\right\} .
$$

We shall give another example and perform numerical computations for $m=4, \quad n=6, \alpha_{1}=0.059, \alpha_{2}=0.063$ and $\alpha_{3}=0.055$. By the similar considerat ions above, we get

$$
\begin{aligned}
& \beta(p)=p^{4}\left[1+a-\left(\begin{array}{c}
6 \\
3
\end{array}\right) p^{3(1+\theta)}\left(1-p^{1+\theta}\right)^{3}{ }_{7+3 \theta}-\frac{4}{+3 \theta}\left\{1+\frac{18(1+\theta)}{(2+\theta) \prod_{j=2}^{4}\{j+2(1+\theta)\}}\right.\right. \\
& \left.+\frac{6(1+\theta)^{2}}{(2+\theta)^{3}(3+\theta)(5+2 \theta)}-\frac{(1+\theta)^{2}(7+2 \theta)}{(2+\theta)^{2}(5+2 \theta)}-\frac{3(1+\theta)^{3}}{(2+\theta)^{2}(3+\theta)}\right\} \\
& -360 p^{2(1+\theta)}\left(1-p^{1+\theta}\right)^{4} \frac{4+3 \theta}{\prod_{j=1}^{4}\{j+2(1+\theta)\}}-4\left(\frac{1}{p}-1\right) p^{(1+\theta)} \frac{6 !(1+\theta)^{6}}{\prod_{k=1}^{6}\{3+k(1+\theta)\}} \\
& -24\left(\frac{1}{p}-1\right)\left(1-p^{1+\theta}\right) p^{5(1+\theta)} \frac{5 !(1+\theta)^{5}}{\prod_{k=1}^{5}\{3+k(1+\theta)\}} \\
& -24\left\{\left(\frac{1}{p}-1\right)-\left(1-p^{2+\theta}\right) /(2+\theta) p^{5(1+\theta)-1} \frac{5 ! 3(1+\theta)^{5}}{(3+\theta) \prod_{k=1}^{5}\{3+k(1+\theta)\}}\right],
\end{aligned}
$$

where $a=2\left(\frac{1}{p}-1\right) p^{5(1+\theta)}\left(12-13 p^{1+\theta}+3 p^{\theta}\right)$.

Denoting $\beta(p) / p^{4}=E(p)$, where $p^{4}$ is the power of the "never pool" test, the value of $E(p)$ seems to show some efficiency of the "sometimes pool" test against the "never pool" test if two tests have the same level of significance. Even not in such favourable circumstance above, $E(p)$ may be an available measure of the power comparision as described later.

We have derived, in the sections 2 and 3 , the values of the size and power of the "sometimes pool" test for various cases in large and small sample. Comparing both results, we may clearly see from Tables and Figuares some similarity between them. In large sample, the relative asymptotic efficiency is generally larger than unity in the neighbourhood of $p=1 / 2$ unless $\alpha_{1}$ is too small. In small sample, there exist some cases where the values of $E(p)(p>1 / 2)$ are larger than unity in spite of smaller size of the test. The examples are the cases $\theta=0.5,0.75$ for $\alpha=$ $4 / 70,9 / 70$ or $\theta=0.5,0.75,1,1.5$ for $\alpha_{1}=37 / 210$ under $p=0.6$. Such inclination is more powerfull when $p=0.8$. It seems to be inadvisable to take the value of $\alpha_{1}$ too large or small. 
TABLE III Comparion of the power (The value of $\mathrm{E}(p)$ )

\begin{tabular}{|c|c|c|c|c|c|c|c|c|}
\hline$p$ & $\alpha_{1} \quad \theta$ & 0 & .25 & .5 & .75 & 1 & 1.5 & 2 \\
\hline 0.5 & $\begin{array}{c}0 \\
4 / 70 \\
9 / 70 \\
37 / 210 \\
5 / 21 \\
32 / 70 \\
66 / 70\end{array}$ & $\begin{array}{r}.875 \\
1.298 \\
1.329 \\
1.400 \\
1.429 \\
1.382 \\
1.048\end{array}$ & $\begin{array}{l}.447 \\
1.042 \\
1.075 \\
1.145 \\
1.168 \\
1.147 \\
1.013\end{array}$ & $\begin{array}{r}.227 \\
.958 \\
.987 \\
1.045 \\
1.063 \\
1.055 \\
1.004\end{array}$ & $\begin{array}{r}.114 \\
.942 \\
.965 \\
1.010 \\
1.022 \\
1.020 \\
1.001\end{array}$ & $\begin{array}{r}.058 \\
.950 \\
.966 \\
.999 \\
1.007 \\
1.008 \\
1.000\end{array}$ & $\begin{array}{l}.015 \\
.974 \\
.982 \\
.997 \\
1.000 \\
1.001 \\
1.000\end{array}$ & $\begin{array}{r}.004 \\
.988 \\
.992 \\
.999 \\
1.000 \\
1.000 \\
1.000\end{array}$ \\
\hline 0.6 & $\begin{array}{c}0 \\
4 / 70 \\
9 / 70 \\
37 / 210 \\
5 / 21 \\
32 / 70 \\
66 / 70\end{array}$ & $\begin{array}{l}1.291 \\
1.567 \\
1.580 \\
1.625 \\
1.636 \\
1.547 \\
1.070\end{array}$ & $\begin{array}{r}.823 \\
1.252 \\
1.268 \\
1.319 \\
1.331 \\
1.269 \\
1.024\end{array}$ & $\begin{array}{l}.520 \\
1.089 \\
1.106 \\
1.156 \\
1.168 \\
1.130 \\
1.009\end{array}$ & $\begin{array}{l}.325 \\
1.013 \\
1.027 \\
1.072 \\
1.083 \\
1.062 \\
1.003\end{array}$ & $\begin{array}{r}.202 \\
.982 \\
.993 \\
1.032 \\
1.040 \\
1.030 \\
1.001\end{array}$ & $\begin{array}{r}.077 \\
.973 \\
.978 \\
1.004 \\
1.009 \\
1.007 \\
1.000\end{array}$ & $\begin{array}{r}.029 \\
.982 \\
.985 \\
.999 \\
1.002 \\
1.002 \\
1.000\end{array}$ \\
\hline 0.8 & $\begin{array}{c}0 \\
4 / 70 \\
9 / 70 \\
37 / 210 \\
5 / 21 \\
32 / 70 \\
66 / 70\end{array}$ & $\begin{array}{l}1.655 \\
1.711 \\
1.702 \\
1.701 \\
1.686 \\
1.572 \\
1.062\end{array}$ & $\begin{array}{l}1.461 \\
1.567 \\
1.553 \\
1.552 \\
1.533 \\
1.412 \\
1.040\end{array}$ & $\begin{array}{l}1.277 \\
1.447 \\
1.427 \\
1.427 \\
1.407 \\
1.293 \\
1.021\end{array}$ & $\begin{array}{l}1.099 \\
1.350 \\
1.325 \\
1.326 \\
1.308 \\
1.207 \\
1.012\end{array}$ & $\begin{array}{r}.953 \\
1.273 \\
1.244 \\
1.246 \\
1.230 \\
1.145 \\
1.007\end{array}$ & $\begin{array}{r}.693 \\
1.167 \\
1.132 \\
1.138 \\
1.126 \\
1.072 \\
1.002\end{array}$ & $\begin{array}{l}.494 \\
1.104 \\
1.067 \\
1.075 \\
1.068 \\
1.036 \\
1.001\end{array}$ \\
\hline
\end{tabular}

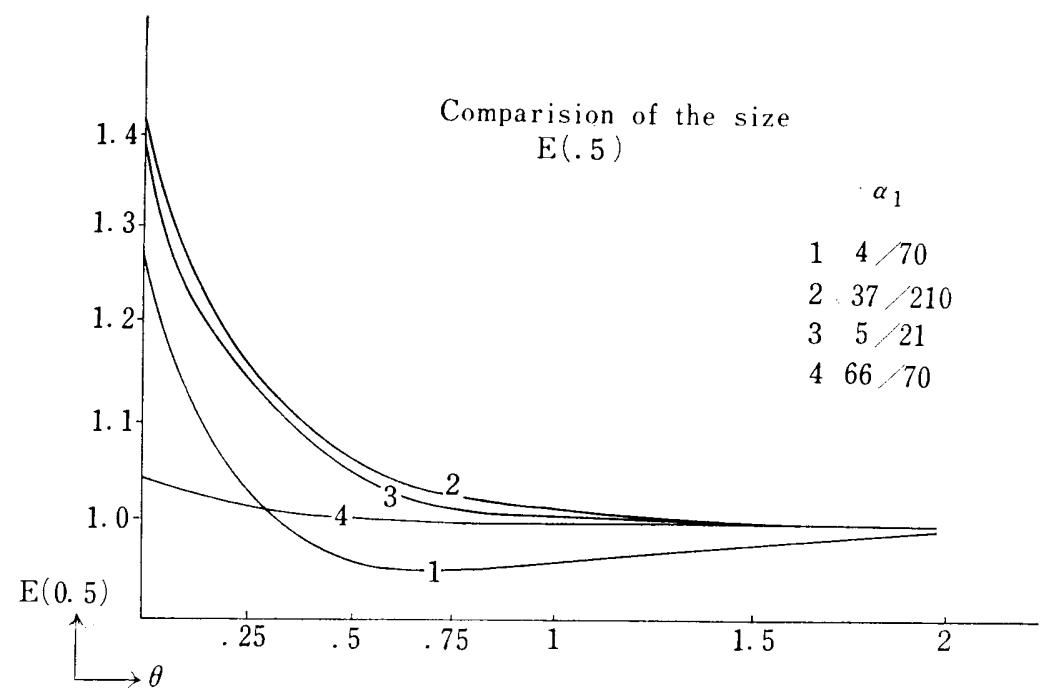

FIGURE III (a)

5. Estimation of median with a preliminary test. In this section, we consider the problem of estimation of the median of $F_{1}(x)$ by "somet. imes pool" procedure. Let the distributions of $X$ and $Y$ be $F_{1}(x)=F(x-\theta)$ and $F_{2}(y)=F\left(y-\theta^{\prime}\right)$ with densities $f(x-\theta)$ and $f\left(y-\theta^{\prime}\right)$ respectively, where $\theta$ and $\theta^{\prime}\left(\theta \leqq \theta^{\prime}\right)$ be the population medians of $X$ and $Y$. Let the sample size be $2 m+1$ and $2 n+1$. Denoting the sample medians by $\tilde{X}$ and $\tilde{Y}$, we define the estimater $\hat{\theta}$ of $\theta$ as follows, 




where $U_{1}$ is a Wilcoxon statistic as in (1) and $N=(2 m+1)+(2 n+1), \lambda=$ $(2 m+1) / N$. Since $V \bar{N}\left(U_{1}-\mu\right)$ is asymptotically equivalent to the statistic

(30) $V_{N}=\frac{1}{V} \frac{1}{\lambda(2 m+1)} \sum_{i=1}^{2 m+1}\left\{\psi_{10}\left(X_{i}\right)-\mu\right\}+\frac{1}{V(1-\lambda)(2 n+1)} \sum_{j=1}^{2 n+1}\left\{\psi_{01}\left(Y_{j}\right)-\mu\right\}$, we shall derive the asymptotic joint distribution of $V_{N}$ and the following $U_{2}$ or $U_{3}$

$$
\begin{aligned}
& U_{2}=\sqrt{2 m+1}(\tilde{X}-\theta) \\
& U_{3}=\sqrt{N}\left\{\xi(\tilde{X}-\theta)+(1-\xi)\left(\tilde{Y}-\theta^{\prime}\right)\right\} .
\end{aligned}
$$

Let the characteristic function of $V_{N}$ and $U_{3}$ be $\varphi\left(t_{1}, t_{2}\right)$, then

$$
\begin{aligned}
\varphi\left(t_{1}, t_{2}\right) & =E\left\{\exp \left(i t_{1} V_{N}+i t_{2} U_{3}\right)\right\} \\
= & E\left[\exp \left\{\frac{i t_{1}}{V^{\prime} \lambda(2 m+1)} \sum_{j=1}^{2 m+1}\left(\psi_{10}\left(X_{j}\right)-\mu\right)+\frac{\xi}{\sqrt{\lambda}} \sqrt{2}-1 i t_{2}(\tilde{X}-\theta)\right\}\right] \\
\times & E\left[\exp \left\{\frac{i t_{1}}{\sqrt{(1-\lambda)(2 n+1)} \sum_{j=1}^{2 n+1}\left(\psi_{01}\left(Y_{j}\right)-\mu\right)+\frac{1-\xi}{\sqrt{1-\lambda}} / 2 n+1} i t_{2}\left(\tilde{Y}-\theta^{\prime}\right)\right\}\right] .
\end{aligned}
$$


Each expected value may be computed by the similar teckniques as Sukhatme [9]. Thus we get the following form

$$
\begin{aligned}
\varphi\left(t_{1}, t_{2}\right) & =\exp \left[-\frac{1}{2}\left\{t_{1}^{2}\left(\begin{array}{c}
\sigma_{11}^{2} \\
\lambda
\end{array}+\frac{\sigma_{12}^{2}}{1-\lambda}\right)+\frac{t_{2}^{2}}{4 f(0)^{2}}\left(\begin{array}{c}
\xi^{2} \\
\lambda
\end{array}+\frac{(1-\xi)^{2}}{1-\lambda}\right)\right.\right. \\
& \left.\left.+2 t_{1} t_{2} \frac{1}{2 f(0)}\left(\frac{\xi}{\lambda}\left(\boldsymbol{m}_{1}^{\prime \prime}-\boldsymbol{m}_{1}^{\prime}\right)+\frac{1-\xi}{1-\lambda}\left(\boldsymbol{m}_{2}^{\prime \prime}-\boldsymbol{m}_{2}^{\prime}\right)\right)\right\}\right]+O(1 / \sqrt{n}),
\end{aligned}
$$

where

$$
\begin{aligned}
& \sigma_{11}^{2}=E \psi_{10}^{2}(X)-\mu^{2}, \sigma_{12}^{2}=E \psi_{01}^{2}(Y)-\mu^{2}, \\
& m_{1}^{\prime}=\int_{-\infty}^{\theta}\left\{\psi_{10}(x)-\mu\right\} d F(x-\theta), m_{1}^{\prime \prime}=\int_{\theta}^{\infty}\left\{\psi_{10}(x)-\mu\right\} d F(x-\theta), \\
& m_{2}^{\prime}=\int_{-\infty}^{\theta^{\prime}}\left\{\psi_{01}(\mathbf{y})-\mu\right\} d F\left(y-\theta^{\prime}\right), m_{2}^{\prime \prime}=\int_{\theta^{\prime}}^{\infty}\left\{\psi_{01}(y)-\mu\right\} d F\left(y-\theta^{\prime}\right), \\
& \psi_{10}(x)=1-F\left(x-\theta^{\prime}\right), \psi_{01}(y)=F(y-\theta) .
\end{aligned}
$$

Thus it follows that $V_{N}$ and $U_{3}$ are jointly asymptotically normally distributed with zero means and the following variances $\sigma_{1}^{2}, \sigma_{3}^{2}$ and correlation $\rho_{3}$. The similar considerations are possible for $V_{N}$ and $U_{2}$. Let the asymptotic density of $v\left(U_{1}-\mu\right)$ and $U_{j}$ be $g_{j}(u, v) \quad(j=2,3)$, then it is given by

$$
g_{j}(u, v)=\frac{1}{2 \pi \sigma_{1} \sigma_{j} V} \overline{\overline{1-\rho_{j}^{2}}} \exp \left[-\frac{1}{2\left(1-\rho_{j}^{2}\right)}\left(\frac{u^{2}}{\sigma_{1}^{2}}+\frac{v^{2}}{\sigma_{j}^{2}}-2 \rho_{j} \frac{u v}{\sigma_{1} \sigma_{j}}\right)\right],
$$

where

$$
\begin{gathered}
\sigma_{1}^{2}=\frac{1}{\lambda}\left(r-\mu^{2}\right)+\frac{1}{1-\lambda}\left(q-\mu^{2}\right), \sigma_{2}^{2}=1 / 4 f(0)^{2}, \sigma_{3}^{2}=\left(\frac{\xi^{2}}{\lambda}+\frac{(1-\xi)^{2}}{1-\lambda}\right) / 4 f(0)^{2}, \\
\rho_{2}=\frac{1}{\sigma_{1} \gamma^{\prime}}\left\{\int_{-\infty}^{0} F(x-h) d F(x)-\int_{0}^{\infty} F(x-h) d F(x)\right\}, h=\theta^{\prime}-\theta, \\
\rho_{3}=\frac{1}{\sigma_{1} \sqrt{\frac{\xi^{2}}{\lambda}}+\frac{(1-\xi)^{2}}{1-\lambda}\left[\frac{\xi}{\lambda}\left\{\int_{-\infty}^{0} F(x-h) d F(x)-\int_{0}^{\infty} F(x-h) d F(x)\right\}\right.} \\
\left.\quad+\frac{1-\xi}{1-\lambda}\left\{\int_{0}^{\infty} F(x+h) d F(x)-\int_{-\infty}^{0} F(x+h) d F(x)\right\}\right] .
\end{gathered}
$$

From (I), (II)

$$
\operatorname{Pr}(\hat{\theta} \leqq w)=\operatorname{Pr}\left(U_{1} \geqq u_{\alpha}, \tilde{X} \leqq w\right)+\operatorname{Pr}\left(U_{1}<u_{\alpha}, \xi \tilde{X}+(1-\xi) \tilde{Y} \leqq w\right) .
$$

Thus the asymptotic c.d.f. $A(w)$ of $\hat{\theta}$ may be derived as follows, 
(35)

$$
\begin{aligned}
A(w) & =\int_{\sqrt{N}\left(u_{\alpha}-\mu\right)}^{\infty} \int_{-\infty}^{\sqrt{2 m+1}(w-\theta)} g_{2}(u, v) d u d v+\int_{-\infty}^{\sqrt{N}\left(u_{\alpha}-\mu\right)} \int_{-\infty}^{\sqrt{N}\left(w-\xi \theta-(1-\xi) \theta^{\prime}\right)} g_{3}(u, v) d u d v \\
& \left.=\int_{-\infty}^{\sqrt{2 m+1}(w-\theta) / \sigma_{2}} \varphi(u)\left\{1-\Phi\left(\frac{k-\rho_{2} u}{1-\rho_{2}^{2}}\right)\right\} d u+\int_{-\infty}^{\sqrt{N}\left(w-\xi \theta-(1-\xi) \theta^{\prime}\right) / \sigma_{3}} \varphi \frac{k-\rho_{3} u}{1-\rho_{3}^{2}}\right) d u,
\end{aligned}
$$

where $k=\jmath / \bar{N}\left(u_{\alpha}-\mu\right) / \sigma_{1}$.

The asymptotic density $a(w)$ may be expressed as follows,

$$
a(w) d w=\varphi\left(w_{2}\right)\left\{1-\Phi\left(\frac{k-\rho_{2} w_{2}}{\sqrt{1-\rho_{2}^{2}}}\right)\right\} d w_{2}+\varphi\left(w_{3}\right) \Phi\left(\frac{k-\rho_{3} w_{3}}{V 1-\rho_{3}^{2}}\right) d w_{3},
$$

where $w_{2}=\sqrt{2 m+1}(w-\theta) / \sigma_{2}, w_{3}=\sqrt{N}\left(w-\xi \theta-(1-\xi) \theta^{\prime}\right) / \sigma_{3}$.

We shall first compute the mean value $\boldsymbol{E}(\hat{\theta})$ from (36),

$$
\begin{aligned}
E(\hat{\theta}) & =\int_{-\infty}^{\infty} w a(w) d w \\
& =\int_{-\infty}^{\infty}\left(\theta+\frac{\sigma_{2}}{\sqrt{2 m+1}} w_{2}\right) \varphi\left(w_{2}\right)\left\{\int_{k}^{\infty} \frac{1}{\sqrt{2 \pi \sqrt{1-\rho_{2}^{2}}}} \exp \left[-\frac{\left(u-\rho_{2} w_{2}\right)^{2}}{2\left(1-\rho_{2}^{2}\right)}\right] d u\right\} d w_{2} \\
& +\int_{-\infty}^{\infty}\left\{\xi \theta+(1-\xi) \theta^{\prime}+\frac{\sigma_{3}}{V \bar{N}} w_{3}\right\} \varphi\left(w_{3}\right)\left\{\int_{-\infty}^{k} \frac{1}{\sqrt{2 \pi \sqrt{1-\rho_{3}^{2}}}} \cdot \exp \left[-\frac{\left(u-\rho_{3} w_{3}\right)^{2}}{2\left(1-\rho_{3}^{2}\right)}\right] d u\right\} d w_{3} \\
& =\theta+(1-\xi)\left(\theta^{\prime}-\theta\right) \Phi(k)+\varphi(k)\left\{\frac{\rho_{2} \sigma_{2}}{\sqrt{2 m+1}}-\frac{\rho_{3} \sigma_{3}}{\sqrt{N}}\right\} \cdot
\end{aligned}
$$

For the mean square error M.S.E.(间,

$$
\begin{aligned}
\text { M.S.E. }(\hat{\theta}) & =\int_{-\infty}^{\infty}(w-\theta)^{2} a(w) d w \\
& =\frac{\sigma_{2}^{2}}{2 m+1}(1-\Phi(k))+\frac{\sigma_{3}^{2}}{N} \Phi(k)+k \varphi(k)\left(\frac{\sigma_{2}^{2} \rho_{2}^{2}}{2 m+1}-\frac{\sigma_{3}^{2} \rho_{3}^{2}}{N}\right) \\
& -\frac{2}{N} \sigma_{3} h \rho_{3}(1-\xi) \varphi(k)+h^{2}(1-\xi)^{2} \Phi(k) .
\end{aligned}
$$

(i) The case $h=h_{0}$ (positive constant). In this case, it holds

$$
\lim _{N \rightarrow \infty} k=-\infty \text {. }
$$

Thus the value of $E(\hat{\theta})$ and $M . S . E .(\hat{\theta})$ respectively tends to $\theta$ and $\sigma_{2}^{2} /(2 m$ $+1)$ where each is the corresponding value of the "never pool" estimator $\tilde{X}$. 
(ii) The case $h=r / / N(r \geq 0)$. It is evident from (37) that

$$
E(\hat{\theta})=\theta+O(1 / \bar{N}) \text {. }
$$

Secondly we may determine the value of $\xi$ minimizing the mean square error from the equality $\frac{d}{d \xi} M . S . E .(\hat{\theta})=0$. The equation may be expressed under $h=0$ as follows,

$$
\left\{\Phi(k)-\frac{3}{4} k \varphi(k)\right\}(\xi-\lambda)=0 .
$$

Thus $\xi=\lambda$ yields the minimum value of the mean square error under $\theta=\theta^{\prime}$. When we adopt $\xi=\lambda$, it follows from (34) that

$$
\rho_{2}=-\frac{1}{2} \sqrt{3(1-\lambda),} \rho_{3}=O(1 / \sqrt{N}), \sigma_{2}^{2}=\sigma_{3}^{2}\left(==\sigma^{2}\right) .
$$

Then (38) may be written as

$$
\text { M.S.E. }(\hat{\theta})=\frac{\sigma_{2}^{2}}{2 m+1}\{1-\dot{\mathcal{H}}(k)\}+\frac{\sigma_{3}^{2}}{N} \Phi(k)+\frac{3}{4 N} \sigma_{2}^{2} k \varphi(k)+o(1 / N) .
$$

The following relation may be easily gained from (38')

$$
\frac{\sigma^{2}}{N}<M . S . E .(\hat{\jmath})<\frac{\sigma^{2}}{2 m+1} .
$$

\section{$\S 5$. Two sample problem.}

5. 1. Test procedure. Let $X_{1}, X_{2}, \cdots, X_{m 1} ; Y_{1}, Y_{2}, \cdots, Y_{m_{2}} ; Z_{1}, Z_{2}, \cdots$, $Z_{m_{3}}$ be three samples from the continuous $c . d . f . F_{1}(x), F_{2}(y)$ and $F_{3}(z)$ respectively. As in the previous sections, we carry out a preliminary test for the main hypothesis

$$
K: F_{1}(x) \equiv F_{2}(x)
$$

against the alternative

$$
K^{\prime}: F_{1}(x)>F_{2}(x) .
$$

The preliminary step is to test the hypothesis $K_{1}$ against the alternative $K_{1}^{\prime}$. Each test is performed by the following Wilcoxon statistics

$$
\begin{aligned}
U_{12} & =\frac{1}{m_{1} m_{2}} \sum_{i=1}^{m_{1}} \sum_{j=1}^{m_{2}} \phi\left(X_{i}, Y_{j}\right), \\
U_{23} & =\frac{1}{m_{2} m_{3}} \sum_{j=1}^{m_{2}} \sum_{i=1}^{m_{3}} \phi\left(Y_{j}, Z_{k}\right), \\
U_{1.23} & =\frac{1}{m_{1}\left(m_{2}+m_{3}\right)}\left\{\sum_{k=1}^{m_{1}} \sum_{j=1}^{m_{2}} \phi\left(X_{i}, Y_{j}\right)+\sum_{i=1}^{m_{1}} \sum_{k=1}^{m_{3}} \phi\left(X_{i}, Z_{k}\right)\right\},
\end{aligned}
$$

where $\phi(x, y)$ is the same function as in (1). 
The means, variances and correlations of these U-statistics may be easily computed as in the previous sections,

$$
\begin{aligned}
& E\left(U_{i j}\right)=\int_{-\infty}^{\infty} F_{i} d F_{j}=\mu_{i j}, E\left(U_{1.23}\right)=\lambda_{23} \mu_{12}+\left(1-\lambda_{23}\right) \mu_{13}=\mu_{1.23} \\
& \operatorname{Var} U_{i j}=\frac{1}{m_{j}}\left(q_{i j}-\mu_{i j}^{2}\right)+\frac{1}{m_{1}}\left(r_{i j}-\mu_{i j}^{2}\right)+o(1 / N)=\sigma_{i j}^{2}, \\
& \operatorname{Var} U_{1.23}=\frac{1}{m_{1} N_{23}^{2}}\left\{m_{2}^{2}\left(r_{12}-\mu_{12}^{2}\right)+m_{1} m_{2}\left(q_{12}-\mu_{12}^{2}\right)+m_{3}^{2}\left(r_{13}-\mu_{13}^{2}\right)\right. \\
& \left.\quad+m_{1} m_{2}\left(q_{13}-\mu_{13}^{2}\right)+2 m_{2} m_{3}\left(\mu_{1.23}-\mu_{12} \cdot \mu_{13}\right)\right\}+o(1 / N)=\sigma_{1.23}^{2}, \\
& \rho\left(U_{23}, U_{12}\right)=\left(\mu_{123}-\mu_{12} \cdot \mu_{23}\right) / m_{2} \sigma_{12} \sigma_{23}=\rho_{12}, \\
& \rho\left(U_{23}, U_{1.23}\right)=\left(\mu_{123}+\mu_{12.3}-\mu_{23} \cdot \mu_{12}-\mu_{23} \cdot \mu_{13}\right) / N_{23} \sigma_{23} \sigma_{1.23}=\rho_{1.23},
\end{aligned}
$$

where

$$
\begin{aligned}
& q_{i j}=\int_{-\infty}^{\infty} F_{i}^{2} d F_{j}, r_{i j}=\int_{-\infty}^{\infty}\left(1-F_{j}\right)^{2} d F_{i}, \mu_{12.3}=\int_{-\infty}^{\infty} F_{1} F_{2} d F_{3}, \\
& \mu_{123}=\int_{-\infty}^{\infty} F_{1}\left(1-F_{3}\right) d F_{2}, \mu_{1.23}=\int_{-\infty}^{\infty}\left(1-F_{2}\right)\left(1-F_{3}\right) d F_{1}, \\
& N_{i j}=m_{i}+m_{j}, \quad N=m_{1}+m_{2}+m_{3}, \lambda_{i j}=m_{i} / N_{i j} .
\end{aligned}
$$

Each critical region of the partial tests is asymptotically given as follows. In the preliminary test,

$$
U_{23} \leqq \frac{1}{2}+\frac{z_{\alpha_{1}}}{\sqrt{N_{23}^{-}}} \sqrt{12\left(\frac{1}{\lambda_{23}}+1-\overline{\lambda_{23}}\right)} \quad\left(=u_{\alpha_{1}}\right),
$$

where $z_{\alpha}$ is defined by (4).

In the main test, it is respectively given by the following

$$
\begin{aligned}
& U_{12} \geqq \frac{1}{2}+\frac{z_{\alpha_{2}}}{\sqrt{N_{12}}} / \frac{1}{12}\left(\frac{1}{\lambda_{12}}+\frac{1}{1-\lambda_{12}}\right)\left(=u_{\alpha_{2}}\right) \text { if (40) holds, } \\
& U_{1.23} \geq \frac{1}{2}+\frac{z_{\alpha_{3}}}{\sqrt{\bar{N}_{23}}} \sqrt{\frac{1}{12 m_{1}}}\left(\lambda_{23} N_{12}+\left(1-\lambda_{23}\right) N_{13}+2 \lambda_{23} m_{3}\right)\left(=u_{\alpha_{3}}\right) \text { if }
\end{aligned}
$$

does not hold.

5.2. The size and power. It may be proved by the similar teckniques as that of section 2 that the joint distribution of our U-statistics are asymptotically normal, then we may get the following expansions. From our test procedure, the size of the test may be expressed as

$$
\operatorname{Pr}\left(U_{23} \geqq u_{\alpha_{1}} \text { and } U_{12} \geqq u_{\alpha_{2}} K\right)+\operatorname{Pr}\left(U_{23}<u_{\alpha_{1}} \text { and } U_{1.23} \geqq u_{\alpha_{3}} K\right) \text {. }
$$


Then

$$
\alpha=\int_{k_{0 . N}}^{\infty} \int_{z_{\alpha 2}}^{\infty} g\left(u, v ; 0,1, \rho_{12.0}\right) d u d v+\int_{-\infty}^{k_{0 N}} \int_{k^{\prime} 0 N}^{\infty} g\left(u, v ; 0,1, \rho_{1.23,0}\right) d u d v,
$$

where

$$
\begin{aligned}
& k_{O N}=\left(u_{\alpha_{1}}-\mu_{23,0}\right) / \sigma_{23,0}, \\
& k_{O N}^{\prime}=\left(u_{\alpha_{3}}-\lambda_{23}^{\lambda_{23}}-\left(1-\lambda_{23}\right) \mu_{13,0}\right) / \\
& \quad \frac{1}{N_{23}} \sqrt{\left.\frac{1}{m_{1}} 1 \frac{1}{12} \overline{m_{2} N_{12}+m_{1}} m_{3}^{2} \sigma_{13.0}^{2}+2 m_{2} m_{3}\left(\mu_{1,23,0}-\frac{\mu_{13,0}}{2}\right)\right\}} .
\end{aligned}
$$

$\mu_{i j, 0}, \sigma_{i j, 0}$ and etc. are the valule under the hypothesis $F_{1}=F_{2}$.

We again assume $F_{j}$ be Lehmann type

$$
F_{3}(x)=F_{2}^{1+\theta_{2}}(x), \theta_{2} \geqq 0 \text {. }
$$

(i) The case $\theta_{2}$ is any constant. Since $\mu_{13}=\mu_{23}=\frac{1}{2}+\delta$ ( $\delta$ is a positive constant), we get $k_{0 N}, k_{0 N}^{\prime} \rightarrow-\infty$ as $N \rightarrow \infty$. Thus we can see from (43) that the value of $\alpha$ tends to $\alpha_{\bar{z}}$ which is the size of the "never pool" test.

(ii) The case $\theta_{2}=r / \gamma \bar{N}(r \geqq 0)$. After some computations, we get



$$
\begin{aligned}
& k_{0}=z_{\alpha_{1}}-\frac{\gamma}{4} \sqrt{\frac{1}{12}\left(\frac{1}{\lambda_{23}}+\frac{1}{1-\lambda_{23}}\right)} \text {, }
\end{aligned}
$$

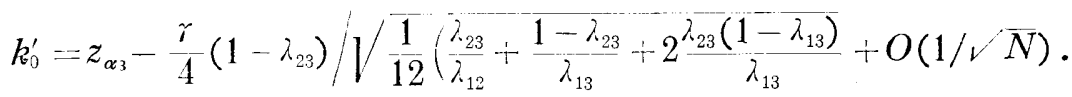

We shall give the results of the case $m_{i}=m$ and $\alpha_{2}=\alpha_{3}=0.0495$ in Table IV and Figure IV.

TABLE IV The size of the test

\begin{tabular}{ccccccc}
\hline$\alpha_{1} \gamma$ & 0 & $v^{\prime} \overline{3}$ & $2 \sqrt{3}$ & $3 \sqrt{3}$ & $4 \sqrt{3}$ & $5 \sqrt{3}$ \\
.0495 & .0471 & .0920 & .1256 & .1150 & .0788 & .0563 \\
.0968 & .0449 & .0811 & .0999 & .0853 & .0621 & .0517 \\
.1587 & .0422 & .0706 & .0804 & .0679 & .0547 & .0502 \\
.2119 & .0400 & .0636 & .0699 & .0604 & .0522 & .0498 \\
.3085 & .0363 & .0538 & .0584 & .0536 & .0503 & .0496 \\
.5 & .0307 & .0440 & .0492 & .0497 & .0496 & .0495 \\
\hline
\end{tabular}

The asymptotic expression $\beta(\theta)$ of the power function may be derived by the similar methods as in the size of the test,

$$
\beta(\theta)=\int_{k_{0 N}}^{\infty} \int_{k_{1 N}}^{\infty} g\left(u, v ; 0,1, \rho_{12}\right) d u d v+\int_{-\infty}^{k_{0 N}} \int_{k_{2 N}}^{\infty} g\left(u, v ; 0,1, \rho_{1.23}\right) d u d v,
$$


where

$$
\begin{aligned}
& k_{1 N}=\left(u_{\alpha_{2}}-\mu_{12}\right) / \sigma_{12}, \\
& k_{2 N}=\left(u_{\alpha_{3}}-\lambda_{23} \mu_{12}-\left(1-\lambda_{23}\right) \mu_{13}\right) / \sigma_{1.23} .
\end{aligned}
$$

We assume the Lehmann type alternative

$$
F_{2}(x)=F_{1}^{1+\theta_{1}}(x), \theta_{1} \geq 0 \text {. }
$$

If the value of $\theta_{1}$ is any positive constant, we get $k_{0 N} \rightarrow k_{0}$ and the other $k_{1 N} \rightarrow-\infty$ as $N \rightarrow \infty$ from (44) and (46). Thus we get

$$
\lim _{N \rightarrow \infty} \beta(\theta)=\int_{k_{0}}^{\infty} \varphi(u) d u+\int_{-\infty}^{k_{0}} \varphi(u) d u=1 .
$$

We have proved that our "sometimes pool" Wilcoxen test has the property of consistency. On the other hand, this property is also true for the " never pool" Wilcoxon test. Secondly we consider the case $\theta_{1}=r_{1} / \sqrt{N_{12}}$, $\gamma_{1} \geq 0$ and $\theta_{2}$ is any positive constant. Since it holds

we get

$$
\lim _{N \rightarrow \infty} k_{0 N}=-\infty, \lim _{N \rightarrow \infty} k_{2 N}=-\infty, \lim _{N \rightarrow \infty} k_{1 N}=z_{\alpha_{2}}-\vartheta \overline{3} r_{1} / 4,
$$

$$
\lim _{N \rightarrow \infty} \beta(\theta)=1-\Phi\left(z_{\alpha_{2}}-\sqrt{3} \gamma_{1} / 4\right) \text {. }
$$

The right hand of (48) expresses the large sample power of the "never pool" Wilcoxon test.

The case $\theta_{1}=r_{1} / \sqrt{ } N_{12}, \theta_{2}=r_{2} /, \overline{N_{23}}, r_{i} \geqq 0$ will be considered from the standpoint of asymptotic relative efficiency in the following paragraph.

5. 3. Asymptotic relative efficiency. Assume $m_{i}=m$ to aviod the complication of computation. From (45), we get

$$
\begin{aligned}
& \beta\left(\theta_{1}\right)=\int_{k_{0}}^{\infty} \varphi(u)\left\{1-\Phi\left(\frac{k_{1}-\rho_{12} u}{V 1-\rho_{12}^{2}}\right)\right\} d u+\Phi\left(k_{0}\right)\left\{1-\Phi\left(k_{2}\right)\right\}, \\
& k_{j}=\lim k_{i N} .
\end{aligned}
$$

On the other hand, the power function of the "never pool" test with the sample sizes $n_{i}$ (assume $n_{i}=n$ ) may be expressed

$$
\begin{aligned}
& \beta_{1}\left(\theta_{1}\right)=1-\Phi(k), \\
& k=\left\{\frac{1}{2}+z_{\alpha} \sqrt{\left.\frac{1}{6 n}-\mu_{12}\right\} / \sqrt{\frac{1}{n}}\left\{\left(r_{12}-\mu_{12}^{2}\right)+\left(q_{12}-\mu_{12}^{2}\right)\right.}\right\} .
\end{aligned}
$$

From the identities

$$
\begin{array}{r}
\left(d \beta\left(\theta_{1}\right) / d \theta_{1}\right)=\frac{V \overline{6 m}}{4} \varphi\left(z_{\alpha_{2}}\right)\left\{1-\Phi\left(\frac{z_{\alpha_{1}}-\frac{\sqrt{3}}{4} \gamma_{2}+\frac{z_{\alpha_{2}}}{\sqrt{3} / 2}}{\sqrt{3}}\right)\right\}+\frac{\sqrt{m}}{\sqrt{2}} \Phi\left(z_{\alpha_{1}}-\frac{\sqrt{3}}{4} \gamma_{2}\right) \\
\cdot \varphi\left(z_{\alpha_{3}}-\gamma_{2} / 4\right)+o(\sqrt{m}),
\end{array}
$$




$$
\left(d \beta_{1}\left(\theta_{1}\right) / d \theta_{1}\right)=\frac{\overline{6 n}}{\theta_{1}=0} \varphi\left(z_{\alpha}\right)+o\left(v^{\prime} n\right),
$$

we get the following asymptotic relative efficiency with regard to the "never pool" test

$$
\begin{aligned}
\mathrm{e}_{s, n}= & \varphi\left(\frac { 1 } { z _ { \alpha } ) ^ { 2 } } \left[\varphi\left(z_{\alpha_{2}}\right)\left\{1-\Phi\left(\frac{z_{\alpha_{1}}-\frac{V}{4} \gamma_{2}+\frac{z_{\alpha_{2}}}{2}}{\sqrt{3} / 2}\right)\right\}\right.\right. \\
& \left.+\frac{2}{\vee} \varphi\left(z_{\alpha_{3}}-r_{2}\right) \oplus\left(z_{\alpha_{1}}-\frac{V}{4} \gamma_{2}\right)\right]^{2} .
\end{aligned}
$$

\begin{tabular}{|c|c|c|c|c|c|c|}
\hline$\alpha_{1} \quad r$ & 0 & $\sqrt{3}$ & $2 \sqrt{3}$ & $3 \sqrt{3}$ & $4 \sqrt{ } 3$ & $5 \sqrt{3}$ \\
\hline .0495 & 1.3093 & 1.2108 & .9646 & .6750 & .6216 & .8155 \\
\hline .0968 & 1.2890 & 1.1557 & .9021 & .7051 & .7618 & .9280 \\
\hline .1587 & 1.2657 & 1.1123 & .8898 & .7846 & .8579 & .9736 \\
\hline .2119 & 1.2441 & 1.0907 & .9007 & .8449 & .8155 & .9876 \\
\hline .3085 & 1.2272 & 1.0891 & .9404 & .9277 & .9280 & .9958 \\
\hline .5 & 1.2128 & 1.0463 & 1.0169 & .9964 & .9736 & .9996 \\
\hline
\end{tabular}

The results of the case $\alpha_{2}=\alpha_{3}=0.0495$ are given in Table $\mathrm{V}$ and Figure $\mathrm{V}$.

TABLE V Relative efficiency $\boldsymbol{e}_{s, n}$



FIGURE IV The size of the test 


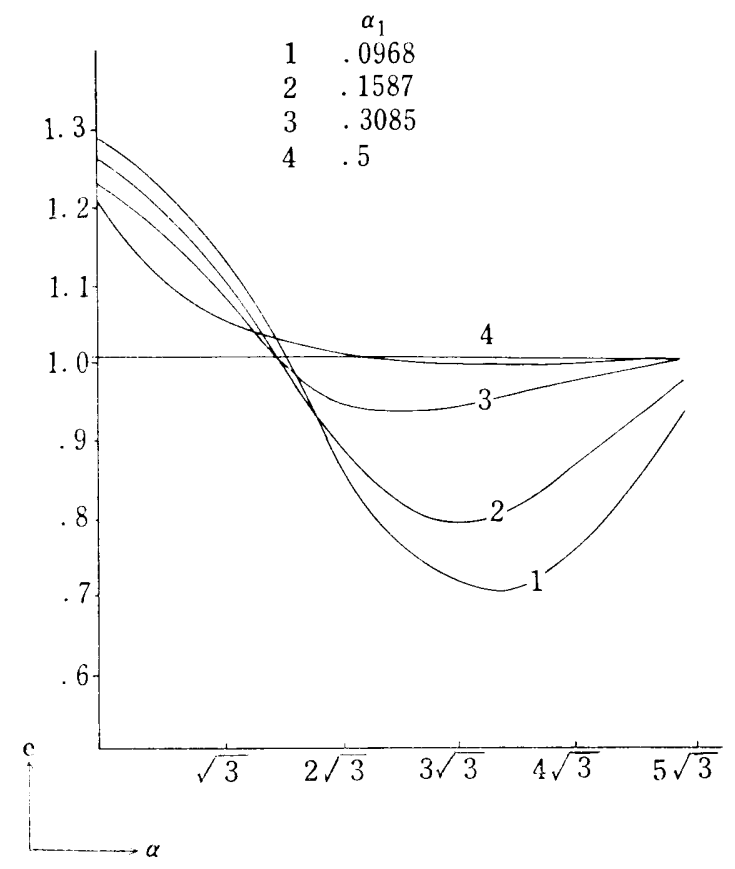

FIGURE V Relative efficiency

\section{4. The scale problem.}

To close this section, we consider the following scale problem. Let the c.d.f. of $X_{i}, Y_{j}, Z_{k}$ be respectively $F(x), F(y / \theta), F\left(z / \theta \theta^{\prime}\right)$ where $\theta, \theta^{\prime} \geq 1$ and $F(0)=1 / 2$. The purpose is to test the hypothesis $\theta=1$ against the alternative $\theta>1$ and we first consider the preliminary test for $\theta^{\prime}=1$. We use the Sukhatme's statistics for the main and preliminary tests,

$$
\begin{aligned}
& T_{23}=\left(m_{2} m_{3}\right)^{-1} \Sigma \Sigma \psi\left(Y_{j}, Z_{k}\right) \\
& T_{12}=\left(m_{1} m_{2}\right)^{-1} \Sigma \Sigma \psi\left(X_{i}, Y_{j}\right) \\
& T_{123}=\lambda_{23} T_{12}+\left(1-\lambda_{23}\right) T_{13},
\end{aligned}
$$

where $\psi(x, y)=1(0)$ if $0<x<y$ or $0>x>y$ (otherwise).

Our test procedure is as f llows. We reject the hypothesis $\theta=1$ when (i) or (ii) holds

$$
\begin{aligned}
& \text { (i) } T_{23} \geq t_{\alpha_{1}} \text { and } T_{12} \geqq t_{\alpha_{2}} \\
& \text { (ii) } T_{23}<t_{\alpha_{1}} \text { and } T_{123} \geqq t_{\alpha_{3}},
\end{aligned}
$$

where

$$
t_{\alpha 1}=\frac{1}{4}+z_{\alpha 1}\left\{48 N_{23} \lambda_{23}\left(1-\lambda_{23}\right)\right\}^{-1 / 2}
$$




$$
\begin{aligned}
& t_{\alpha_{2}}=\frac{1}{4}+z_{\alpha_{2}}\left\{48 N_{12} \lambda_{12}\left(1-\lambda_{12}\right)\right\}^{-1 / 2} \\
& t_{\alpha_{3}}=\frac{1}{4}+z_{\alpha_{3}}\left\{\begin{array}{c}
1 \\
48 N_{23}
\end{array}\left(\frac{\lambda_{23}}{\lambda_{12}}+\frac{1-\lambda_{23}}{\lambda_{13}}+\frac{2 \lambda_{23}\left(1-\lambda_{13}\right)}{\lambda_{13}}\right)\right\}^{1 / 2} .
\end{aligned}
$$

Then the size $\alpha$ of the test is given from (53) by the following

$$
\alpha=\operatorname{Pr}\left(T_{23} \geq t_{\alpha_{1}}, T_{12} \geqq t_{\alpha_{2}} \theta=1\right)+\operatorname{Pr}\left(T_{23}<t_{\alpha_{1}}, T_{123} \geq t_{\alpha_{3}} \theta=1\right) \text {. }
$$

Now we assume that $\theta^{\prime}=1+\delta$ ( $\delta$ is any positive constant), then the hypothesis $\theta^{\prime}=1$ is almost certainly rejected in the preliminary test by the similarly reason as the previous 5.2. Therefore our procedure is equivalent to the "never pool" test. Thus we consider only the case of $\theta^{\prime}=1+r / \mathrm{V} N_{23}$. In the case of $\theta=1$ and $\theta^{\prime}=1+\gamma / \mathrm{\gamma} N_{23}^{-}$, the following are easy,

$$
\begin{aligned}
& E\left(T_{12}\right)=1 / 4 \\
& E\left(T_{23}\right)=1 / 4+\gamma k / \vee N_{23}^{-}, k=\int_{0}^{\infty} x f^{2}(x) d x-\int_{-\infty}^{0} x f^{2}(x) d x \\
& E\left(T_{123}\right)=1 / 4+\left(1-\lambda_{23}\right) r k / \sqrt{N_{23}^{-}} \\
& \operatorname{Var}\left(T_{i j /} / N_{i j}\right)=\sigma_{i j}^{2}=\left\{48 \lambda_{i j}\left(1-\lambda_{i j}\right)\right\}^{-1} \\
& \operatorname{Var}\left(\mathrm{V} / \bar{N}_{23} T_{123}\right)=\sigma_{123}^{2}=48^{-1}\left\{\frac{\lambda_{23}}{\lambda_{13}}+\frac{1-\lambda_{23}}{\lambda_{13}}+\frac{2 \lambda_{23}\left(1-\lambda_{13}\right)}{\lambda_{13}}\right\} \\
& \operatorname{Corr} .\left(T_{23}, T_{12}\right)=\rho=-\sqrt{\lambda_{13}}\left(1-\lambda_{23}\right) \\
& \operatorname{Corr} .\left(T_{23}, T_{123}\right)=O(1 / \sqrt{N}) .
\end{aligned}
$$

Thus we may asymptotically express the size $\alpha$ by using (54)

$$
\begin{aligned}
\alpha= & \int_{z_{\alpha_{1}-\gamma k / \sigma-3}}^{\infty} \int_{z_{\alpha_{2}}}^{\infty} g\left(x, y ; 0,0 ; 1,1 ;-\sqrt{\lambda_{12}}\left(\overline{1-\lambda_{23}}\right)\right) d x d y \\
& +\Phi\left(z_{\alpha_{1}}-\gamma k / \sigma_{23}\right)\left[1-\Phi\left\{z_{\alpha_{3}}-\left(1-\lambda_{23}\right) \gamma k / \sigma_{123}\right\}\right] .
\end{aligned}
$$

Under the assumptions $f(x)=N(0,1), \lambda_{i j}=1 / 2$, we get the values

$$
k=1 / 2 \pi, \rho=-1 / 2, \sigma_{i j}^{2}=1 / 12, \sigma_{123}^{2}=1 / 16 .
$$

Then,

$$
\alpha=\int_{z_{\alpha_{1}-\gamma} \frac{\sqrt{3}}{\pi}}^{\infty} \int_{z \alpha_{2}}^{\infty} g\left(x, y ; 0,0 ; 1,1 ;-\frac{1}{2}\right) d x d y+\Phi\left(z_{\alpha_{1}}-\gamma \frac{\gamma}{\pi}\right)\left\{1-\Phi\left(z_{\alpha_{3}}-\frac{r}{\pi}\right)\right\} .
$$

This form is identical to that of the size in 5.2 by letting $r=\frac{\pi}{4} r^{\prime}$ and hence its behaviour is similar as the Table IV. By the similar considerat- 
ions as 5.3, we may derive the asymptotic efficiency with regard to the " never pool" Sukhatme's test,

$$
\begin{aligned}
e_{s, n}=\frac{1}{\varphi\left(z_{\alpha}\right)^{2}}\left[\varphi\left(z_{\alpha_{2}}\right)\left\{1-\Phi\left(\frac{z_{\alpha_{1}}-\sqrt{ } \gamma / \pi+z_{\alpha_{2}} / 2}{\sqrt{ } / 2}\right)\right\}\right. \\
\left.+\frac{2}{\sqrt{ } 3} \varphi\left(z_{\alpha_{3}}-\gamma / \pi\right) \Phi\left(z_{\alpha_{1}}-v^{\prime} 3 r / \pi\right)\right]^{2} .
\end{aligned}
$$

\section{\$. Some discussions.}

Though we have used Wilcoxon statistics for two sample tests for location, we have no reasons for its necessity. We may also use another statistics to test the hypothesis $F_{i}=F_{j}$. We consider the more general forms of rank order statistics

$$
m_{i} T_{i j}=\sum_{k=1}^{N_{i i}} E_{N_{i j, k}} z_{N_{i j}, k},
$$

where $z_{N_{i j}, k}$ is the random variable which takes 1 or 0 according the $k$ th smallest in the combined sample is from $F_{i}$ or $F_{j}$ and $E_{N, k}$ are given constants. It is well-known that (57) contains as the special cases the statistics, i.e. Wilcoxon, Hoeffiding's c-statistic [4] and etc. The asymptotic normality of $T$ has been proved under some regularity assumptions by Chernoff-Savage [1]. We shall show the asymptotic joint normality of two $T$ statistic (similar for more than two) under the same assumptions as in [1]. In fact, $T_{i j}$ may be rewritten

$$
\begin{aligned}
T_{i j}=\int_{-\infty}^{\infty} J\left\{H _ { i j } ( x ) \left\{d F_{i}(x)+\left(1-\lambda_{i j}\right)\left[\frac{1}{m_{i}} \sum_{k}^{m_{i}}\left\{B_{i j}\left(X_{k}\right)-E B_{i j}\left(X_{k}\right)\right\}\right.\right.\right. \\
\left.-\frac{1}{m_{j}} \sum_{k}^{m j}\left\{B_{i j}^{*}\left(Y_{k}\right)-E B_{i j}^{*}\left(Y_{k}\right)\right\}\right]+o\left(1 / N_{i j}\right),
\end{aligned}
$$

where

$$
\begin{aligned}
& H_{i j}(x)=\lambda_{i j} F_{i}(x)+\left(1-\lambda_{i j}\right) F_{j}(x) \\
& J_{N_{i j}}\left(k / N_{i j}\right)=E_{N_{i j}, k}, \lim _{N \rightarrow \infty} J_{N}(t)=J(t), 0<t<1 \\
& B_{i j}(x)=\int_{x_{0}}^{x} J^{\prime}\left\{H_{i j}(x)\right\} d F_{j}(x), B_{i j}^{*}(x)=\int_{x_{0}}^{x} J^{\prime}\left\{H_{i j}(x)\right\} d F_{i}(x)
\end{aligned}
$$

Applying the Central Limit Theorem for the random vectors

$$
W_{k}=\left\{B_{12}\left(X_{k}\right)-E B_{12}\left(X_{k}\right), B_{13}\left(X_{k}\right)-E B_{13}\left(X_{k}\right)\right\} \quad k=1, \cdots \cdots, m_{1}
$$

, then $\sum W_{k} / \sqrt{m_{1}}$ distributes following the asymptotic bivariate normal distribution under some regurarity conditions. The fact leads us to the 
applications of such statistics for our "sometimes pool" procedure. We may also prove that $T_{12}$ and $\hat{\theta}$ are jointly asymptotically normally distributed. Let the characteristic function of $\bar{N}\left(T_{12}-a\right) /(1-i)$ and $U_{3}$ in (31) be $\varphi\left(t_{1}, t_{2}\right)$ where $a=\int_{-\infty}^{\infty} J\left\{H_{12}(x)\right\} d F_{1}(x)$, then we get

$$
\begin{aligned}
\varphi\left(t_{1}, t_{2}\right) & =E\left[\exp \left\{\frac{i t_{1}}{V m_{1}} \sum\{B(X)-E B(X)\}+\frac{\xi}{V /} \sqrt{m_{1}} i t_{2}(\tilde{X}-\theta)\right\}\right] \\
& \times E\left[\exp \left\{\frac{-i t_{1}}{V(1-\lambda) m_{2}} \sum\left\{B^{*}(X)-E B^{*}(X)\right\}+\frac{1-\xi}{\sqrt{1-\lambda}} V^{\prime} \overline{m_{2}} i t_{2}(Y-\theta)\right\}\right] .
\end{aligned}
$$

The same tecknique as Section 4 shows the joint asymptotic normality.

\section{References}

[1] Chernoff, H. and SaVage, I. R. (1958). Asymptotic normality and effciency of certain nonparametric test statistics. Ann. Math. Statist. 29 972-994.

[2] Dwaqs, M. (1956). The large-sample power of rank order tests in the two-sample problem. Ann. Math. Statist. 27 352-374.

[3] Hoeffoing, W. (1948). A class of statistics with asymptotically normal distribution. Ann. Math. Statist. $19293-325$.

[4] Hoffrding, W. (1951). "Optimum" nonparametric tests. Proc. Second Berkeley Sympo. on Math. Stat, and Prob. Univ. Calif. Press 83-92.

[5] Kitalawa, T. (1950). Successive process of statistical inferences. Mem. Faculty of Scie. Kyushu Univ. Ser. A $139-180$.

[6] Lemmann, E. L. (1953). The power of rank tests. Ann. Math. Statist. $2423-43$.

[7] Owen, D. B. (1956). Tables for computing bivariate normal probabilities. Ann. Math. Statist. 27 1075-1090.

[8] PAUL, A. E. (1950). On a preliminary test for pooling mean squares in the analysis of variance. Ann Math Statist. 21 539-556.

[9] Surhatme, B. V. (1957). Joint asymptotic distribution of the median and a U-statistic. JRSS, Series B 19 144-148. 